\title{
Imaging performance and clinical value of contrast-enhanced ultrasonography and computed tomography in the diagnosis of liver cancer
}

\author{
BOLIN SUN ${ }^{1 *}$, YONGBIN LV ${ }^{2 *}$, DONG XING ${ }^{2}$ and JIANLIN LI' \\ ${ }^{1}$ Interventional Therapy Ward and ${ }^{2}$ Department of Radiology, Yuhuangding Hospital, Yantai, Shandong 264000, P.R. China
}

Received December 21, 2017; Accepted February 20, 2018

DOI: $10.3892 / \mathrm{ol} .2018 .8281$

\begin{abstract}
The imaging performance and clinical value of contrast-enhanced ultrasonography (CEUS) combined with $\mathrm{CT}$ in diagnosis of liver cancer were investigated. A total of 426 liver cancer patients treated in Yuhuangding Hospital (Yantai, China) from February 2011 to May 2016 were selected. Among them, 222 patients underwent CEUS examination, 204 patients underwent CT examination, and 102 patients underwent CEUS combined with CT examination. Sensitivity and specificity of the three methods were examined. CT showed a low density in $92.6 \%$ of patients (189 patients) and a high-low hybrid density in $6.4 \%$ (13 patients), with calcification seen in $2.5 \%$ of patients (5 patients) and bleeding in $3.4 \%$ (7 patients). Lesions: $76.5 \%$ (156 cases) of patients with multiple type, $23.5 \%$ (48 cases) with single type. CEUS showed overall enhancement in $53.2 \%$ patients (118 cases), heterogenous enhancement in $16.7 \%$ (37 cases), peripheral enhancement in $18.9 \%$ of patients (42 cases), necrosis of liquefaction in $11.3 \%$ (25 cases). In $65.3 \%$ (145 cases) of patients, the portal venous phase and the delayed phase showed a low enhancement, while $34.7 \%$ (77 cases) showed no enhancement. The sensitivity and specificity rates of CEUS combined with CT detection of liver cancer were $87.8,88.2$ and $94.1 \%$, respectively. The ROC curve analysis showed that the sensitivity and specificity of CEUS in the diagnosis of liver cancer were 76.8 and $78.9 \%$, respectively. The sensitivity and specificity of CT were 81.2 and $85.5 \%$, respectively. The sensitivity and specificity of CEUS combined with CT were 90.4 and $92.7 \%$, respectively. CEUS combined with CT detection can make-up for the deficiencies of each other and effectively improve the coincidence
\end{abstract}

Correspondence to: Dr Jianlin Li, Department of Radiology, Yuhuangding Hospital, 20 East Yuhuangding Road, Yantai, Shandong 264000, P.R. China

E-mail: jrd5ce@163.com

*Contributed equally

Key words: contrast-enhanced ultrasonography, computed tomography, liver cancer, combined diagnosis, clinical value rate of liver cancer diagnosis, which can be used as an effective examination method for the diagnosis of liver cancer.

\section{Introduction}

Liver cancer is one of the most common malignant tumors in the digestive system. The mortality rate is relatively high, and the early stage of liver cancer can be asymptomatic. Once the symptoms appear, most of the patients have advanced into the late stage, which seriously affects the treatment and prognosis of the patients $(1,2)$. Therefore, early diagnosis and treatment of liver cancer is a crucial step. China is a country with high hepatitis B incidence, therefore, many liver cancers are derived from the development of hepatitis B cirrhosis, and hepatitis $\mathrm{C}$ patients are gradually increasing, leading directly to the huge number of liver cancer patients in recent years, therefore, the diagnosis and treatment of liver cancer is very important to our medical system (3). Recently, the imaging diagnosis of liver cancer in many hospitals is still mainly based on the characteristics of blood supply, and contrast-enhanced ultrasonography (CEUS) which is a widely-used technology developed in recent years providing real-time monitoring of the dynamic distribution of blood vessels of liver cancer patients revealing the hemodynamic characteristics of liver cancer patients (4). However, CEUS has low repeatability and limitations of detection time, and it can only observe one lesion at one time. As a noninvasive imaging technique, $\mathrm{CT}$ also has a good diagnostic value in patients with liver cancer (5). However, CT cannot accurately detect early lesions with small or limited density changes and early lesions in cellular levels, and it is extremely easy to produce artifacts due to movement or metal. Therefore, we considered that combining CEUS and CT in the diagnosis of liver cancer can make up for the deficiencies of each other, and through the CEUS and CT imaging results of 426 cases of liver cancer patients, the imaging performance and the value of diagnosis of liver cancer were analyzed.

\section{Patients and methods}

General information. A total of 426 patients with liver cancer confirmed by pathology and admitted to Yuhuangding Hospital (Yantai, China) from February 2011 to May 2016 were selected. There were 336 males and 90 females, with an average age of $56.3 \pm 10.5$ years. Among them 195 cases 
Table I. Basic information of 426 patients (n, \%).

\begin{tabular}{|c|c|c|c|c|c|}
\hline Parameters & CEUS group $(\mathrm{n}=222)$ & CT group $(n=204)$ & CEUS+CT group $(n=102)$ & F-value & P-value \\
\hline Age (years) & & & & 2.350 & 0.293 \\
\hline$<59$ & $124(55.9)$ & $119(58.3)$ & $61(59.8)$ & & \\
\hline$\geq 59$ & $98(44.1)$ & $85(41.7)$ & $41(40.2)$ & & \\
\hline Sex & & & & 3.280 & 0.448 \\
\hline Male & $184(82.9)$ & $167(81.9)$ & $87(85.3)$ & & \\
\hline Female & $38(17.1)$ & $37(18.1)$ & $15(14.7)$ & & \\
\hline Ethnicity & & & & 3.185 & 0.485 \\
\hline Han & 214 (96.6) & $199(97.5)$ & 99 (97.1) & & \\
\hline Others & $8(3.6)$ & $5(2.5)$ & $3(2.9)$ & & \\
\hline Marital status & & & & 2.212 & 0.410 \\
\hline Married & 207 (93.2) & $184(92.2)$ & $89(87.3)$ & & \\
\hline Unmarried & $11(5.0)$ & $18(8.8)$ & $10(9.8)$ & & \\
\hline Widowed & $4(1.8)$ & $2(1.0)$ & $3(2.9)$ & & \\
\hline Living area & & & & 3.038 & 0.455 \\
\hline Countryside & $134(60.4)$ & $119(58.3)$ & $64(62.7)$ & & \\
\hline City & 88 (39.6) & $85(41.7)$ & $38(37.3)$ & & \\
\hline Smoking index & & & & 3.074 & 0.332 \\
\hline$<400$ & $61(27.5)$ & $59(28.9)$ & $24(23.5)$ & & \\
\hline$\geq 400-<800$ & $77(34.7)$ & $69(33.8)$ & $37(36.3)$ & & \\
\hline$\geq 800$ & $84(37.8)$ & $76(37.3)$ & $41(40.2)$ & & \\
\hline Drinking & & & & 2.584 & 0.310 \\
\hline $\begin{array}{l}\text { No drinking or } \\
\text { occasionally }\end{array}$ & $74(33.3)$ & $51(25.0)$ & $22(21.6)$ & & \\
\hline Often & $148(66.7)$ & $153(75.0)$ & $80(78.4)$ & & \\
\hline
\end{tabular}

CEUS, contrast-enhanced ultrasonography; CT, computed tomography.

were benign and 231 cases were malignant. Two hundred and twenty-two patients underwent CEUS examination, 204 patients underwent CT examination and 102 patients underwent CEUS combined with CT examination. Inclusion criteria: Forty-five years of age and above, liver cancer patients confirmed by histology or cytology, no previous diagnosis of liver cancer, patients with no family history of genetic disease. Exclusion criteria: Critically ill patients, patients with incomplete limbs, patients not cooperating with the relevant examination, patients suffering from cancer other than liver cancer. All the patients included in this study or their families signed informed consent. The relevant study was carried out under the approval of the Ethics Committee of Yuhuangding Hospital, and the patients were informed and they signed a formal authorization.

Instruments and methods. Patients fasted for $5 \mathrm{~h}$ before the test, and the corresponding allergy test was performed, Sequoia 512 Siemens diagnostic ultrasound system with real-time contrast pulse sequence CEUS technology was adopted, contrast agent was Sono Vue (GE, USA). Sono Vue was diluted with $5 \mathrm{ml}$ of normal saline, and $2.4 \mathrm{ml}$ of diluted contrast medium was drawn. The bolus was injected through the anterior left elbow vein followed by bolus injection of $5 \mathrm{ml}$ of normal saline. The injection was simultaneously timed to observe the liver lesions. The time of contrast imaging was 5-7 min. The LightSpeed 16-slice spiral CT manufactured by GE Healthcare Life Sciences (Waukesha, WI, USA), was used and high-quality scanning mode was applied. The scanning parameters were $7.5 \mathrm{~mm}$ layer thickness, $1.25 \mathrm{~mm}$ resolution, $1.375 \mathrm{I}$ pitch, $120 \mathrm{kV}$ voltage and $280 \mathrm{~mA}$ tube current.

Image analysis. Combined with the clinical data of patients, the diagnostic accuracy of the three diagnostic methods were evaluated by taking pathological sections as the gold standard of diagnosis. The characteristics of CEUS and CT scanning of lesions were observed, the focus of observation was the lesion density, number, and marginal conditions. The CEUS and CT images of all patients were analyzed by double blind method and analyzed by four experienced imaging physicians, the clinical values were also observed.

Statistical analysis. Using SPSS 22.0 data analysis system (Boyi Information Technology Co., Ltd., Guangzhou, China) for data analysis, the enumeration data are expressed as percentage, the comparison among groups used Chi-square test, the sensitivity and specificity of diagnosis was analyzed using ROC curve analysis. 
Table II. Clinical symptoms of 426 patients (n, \%).

\begin{tabular}{|c|c|c|c|c|c|}
\hline Parameters & CEUS group $(n=222)$ & CT group $(n=204)$ & CEUS+CT group $(n=102)$ & F-value & P-value \\
\hline Pathological types & & & & 3.249 & 0.327 \\
\hline Block & $19(8.6)$ & $17(8.3)$ & $7(6.9)$ & & \\
\hline Large block & $39(17.6)$ & $41(20.1)$ & $16(15.7)$ & & \\
\hline Nodular & $81(36.5)$ & $74(36.3)$ & $43(42.2)$ & & \\
\hline Diffuse & $36(16.2)$ & $29(14.2)$ & $10(9.8)$ & & \\
\hline Small cancer & $6(2.7)$ & $5(2.5)$ & $3(2.9)$ & & \\
\hline $\mathrm{T}$ staging & & & & 3.467 & 0.458 \\
\hline TX & $1(0.5)$ & $0(0.0)$ & $1(1.0)$ & & \\
\hline T0 & $8(3.6)$ & $7(3.4)$ & $2(2.0)$ & & \\
\hline $\mathrm{T} 1$ & $25(11.3)$ & $19(9.3)$ & $14(13.7)$ & & \\
\hline $\mathrm{T} 2$ & $48(21.6)$ & $40(19.6)$ & $23(22.5)$ & & \\
\hline $\mathrm{T} 3$ & $62(27.9)$ & $50(24.5)$ & $22(21.6)$ & & \\
\hline $\mathrm{T} 4$ & $46(20.7)$ & $37(18.1)$ & $21(20.6)$ & & \\
\hline $\mathrm{N}$ staging & & & & 2.277 & 0.390 \\
\hline NX & $5(2.3)$ & $3(1.5)$ & $3(2.9)$ & & \\
\hline No & $97(43.7)$ & $83(40.7)$ & $41(40.2)$ & & \\
\hline N1 & $85(38.3)$ & $75(36.8)$ & $34(33.3)$ & & \\
\hline M staging & & & & 2.915 & 0.379 \\
\hline MX & $7(3.2)$ & $5(2.5)$ & $2(2.0)$ & & \\
\hline M0 & $90(40.5)$ & $78(38.2)$ & $33(32.4)$ & & \\
\hline M1 & $92(41.4)$ & $79(38.7)$ & $40(39.2)$ & & \\
\hline Clinical manifestations & & & & 3.391 & 0.426 \\
\hline Fever and edema & $42(18.9)$ & $39(19.1)$ & $22(21.6)$ & & \\
\hline Weight loss & $56(25.2)$ & $47(23.0)$ & $28(27.5)$ & & \\
\hline Bloating, nausea, vomiting & $39(17.6)$ & $27(13.2)$ & $17(16.7)$ & & \\
\hline $\begin{array}{l}\text { Right upper quadrant } \\
\text { dull pain }\end{array}$ & $74(33.3)$ & $66(32.4)$ & $39(38.2)$ & & \\
\hline Liver pain & $51(23.0)$ & $59(28.9)$ & $34(33.3)$ & & \\
\hline Jaundice & $86(38.7)$ & $74(36.3)$ & $44(43.1)$ & & \\
\hline Dyspnea & $124(55.9)$ & $110(53.9)$ & $56(54.9)$ & & \\
\hline Cavity effusion & $154(69.4)$ & $139(68.1)$ & $64(62.7)$ & & \\
\hline
\end{tabular}

CEUS, contrast-enhanced ultrasonography; CT, computed tomography.

\section{Results}

Clinical data of patients. According to the clinical data of patients, there was no significant difference in age, sex, ethnicity, marital status, living area, smoking index, alcohol drinking and pathological classification, TNM staging and clinical manifestations among patients receiving CEUS, CT and the two combined detection ( $p>0.05)$, further reducing the experimental error (Tables I and II).

$C T$ results. The features of plain scan in 204 patients undergoing CT scan were: Low density in $92.6 \%$ of patients (189 cases), low and high density in 6.4\% (13 cases), calcification in $2.5 \%$ of patients ( 5 cases), and $3.4 \%$ (7 cases) showed bleeding. Lesions: $76.5 \%$ (156 cases) of patients with multiple type, $23.5 \%$ (48 cases) with single type. In $80.9 \%$ (165 cases), the boundary of the lesions was blurred, and in $19.1 \%$ (39 cases) the boundaries of the lesions were clear. Dynamic contrastenhanced features in 204 patients: 56.4\% (115 cases) patients showed progressive enhancement. In $22.1 \%$ (45 cases) patients showed no significant enhancement. In $11.8 \%$ (24 patients), the patients showed marginal anadem-like enhancement. In $9.8 \%$ (20 cases) of patients fast-in, fast-out type was seen. Dynamic enhanced scan feature classification criteria are shown in Table III.

CEUS imaging results. In 222 patients undergoing CEUS, the imaging results were as follows: $53.2 \%$ (118 patients) showed overall enhancement, $16.7 \%$ (37 patients) showed heterogeneous enhancement, $18.9 \%$ (42 patients) showed peripheral enhancement, and $11.3 \%$ (25 cases) patients showed necrosis with liquefaction. In $65.3 \%$ (145 cases) patients, the portal venous phase and the delayed phase showed a low enhancement, while $34.7 \%$ (77 cases) showed no enhancement. 
Table III. Dynamic enhanced scanning feature classification criteria.

\begin{tabular}{|c|c|}
\hline Classification & Criteria \\
\hline $\begin{array}{l}\text { Progressive } \\
\text { enhancement }\end{array}$ & $\begin{array}{l}\text { Mild enhancement in the margin of } \\
\text { the lesion in arterial phase, obvious } \\
\text { enhancement in portal venous phase }\end{array}$ \\
\hline $\begin{array}{l}\text { No significant } \\
\text { enhancement }\end{array}$ & $\begin{array}{l}\text { No obvious enhancement in the } \\
\text { lesions or visible nodular enhancemen } \\
\text { at the edge }\end{array}$ \\
\hline $\begin{array}{l}\text { Marginal anadem-like } \\
\text { enhancement }\end{array}$ & $\begin{array}{l}\text { Visible anadem-like lesions } \\
\text { observed at the edge of the lesions }\end{array}$ \\
\hline $\begin{array}{l}\text { Fast-in, fast-out } \\
\text { enhancement }\end{array}$ & $\begin{array}{l}\text { Obvious enhancement in arterial } \\
\text { phase, lesion enhancement was } \\
\text { significantly reduced in portal } \\
\text { venous phase and delayed phase }\end{array}$ \\
\hline
\end{tabular}

Table IV. Accuracy of three diagnostic methods (n, \%).

\begin{tabular}{lccc}
\hline Methods & Diagnosed & Misdiagnosed & Accuracy (\%) \\
\hline CEUS & $195(87.8)$ & $27(12.2)$ & 87.8 \\
CT & $180(88.2)$ & $24(11.8)$ & 88.2 \\
CEUS+CT & $96(94.1)$ & $6(5.9)$ & 94.1 \\
$\chi^{2}$ & 12.54 & 13.47 & 13.87 \\
P-value & 0.041 & 0.037 & 0.032 \\
\hline
\end{tabular}

CEUS, contrast-enhanced ultrasonography; CT, computed tomography.

Table V. ROC curve analysis.

\begin{tabular}{lcccc}
\hline Methods & AUC & $95 \%$ CI & $\begin{array}{c}\text { Sensitivity } \\
(\%)\end{array}$ & $\begin{array}{c}\text { Specificity } \\
(\%)\end{array}$ \\
\hline CEUS & 0.724 & $0.211-2.534$ & 76.8 & 78.9 \\
CT & 0.784 & $0.617-1.232$ & 81.2 & 85.5 \\
CEUS+CT & 0.846 & $0.146-4.643$ & 90.4 & 92.7 \\
$\chi^{2}$ & & & 13.51 & 12.74 \\
P-value & & & 0.037 & 0.041 \\
\hline
\end{tabular}

CI, confidence interval; CEUS, contrast-enhanced ultrasonography; CT, computed tomography.

Diagnostic accuracy. Of the 222 patients who underwent CEUS examination, 195 were diagnosed with liver cancer, with a diagnostic accuracy of $87.8 \%$. Of the 204 patients who underwent CT examination, 180 were diagnosed with liver cancer and the diagnostic accuracy was $88.2 \%$. Of the 102 patients who underwent CEUS combined CT examination, 96 cases were diagnosed as liver cancer, the diagnostic accuracy was $94.1 \%$. The accuracy of joint detection was better than single detection $(\mathrm{p}<0.05$; Table IV).

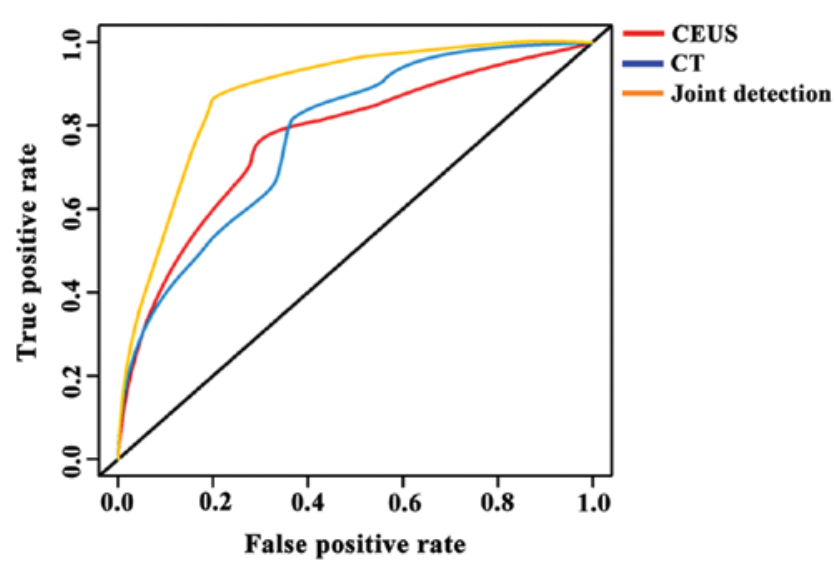

Figure 1. ROC curve analysis. The sensitivity and specificity of CEUS in the diagnosis of liver cancer were 76.8 and $78.9 \%$, respectively. The sensitivity and specificity of CT were 81.2 and $85.5 \%$, respectively. The sensitivity and specificity of CEUS and CT were 90.4 and $92.7 \%$, respectively. CEUS, contrast-enhanced ultrasonography; CT, computed tomography.

ROC curve analysis. The ROC curve showed that the AUC of CEUS was 0.724 (95\% CI, 0.211-2.534); the AUC of CT was 0.784 (95\% CI, 0.617-1.232); the AUC of CEUS combined with CT was 0.846 (95\% CI, 0.146-4.643). The sensitivity and specificity of CEUS in the diagnosis of liver cancer were 76.8 and $78.9 \%$, respectively. The sensitivity and specificity of CT were 81.2 and $85.5 \%$, respectively. The sensitivity and specificity of CEUS and CT were 90.4 and $92.7 \%$, respectively. The specificity and sensitivity of combined detection were better than single detection $(\mathrm{p}<0.05)$ (Table V; Fig. 1).

\section{Discussion}

Liver cancer is a highly malignant disease with high incidence among the malignant tumors, early diagnosis and treatment of the disease is the most important and effective way to improve the prognosis of patients clinically (6). The early stage of liver cancer has no obvious clinical features, the rough assessment is mainly through the imaging method (7). Nowadays in clinic, MRI, CT, CEUS and other imaging methods can achieve a more accurate diagnosis of liver cancer (8). According to studies by Fischer et al (9), $\mathrm{CT}$ is the most accurate diagnosis of liver cancer. While Mishima et al (10) asserted that CEUS was more effective in showing the performance of liver cancer lesions, which is more widely recommended in the early diagnosis of liver cancer clinically. The imaging method of single examination, though short in time and radiation damage to patients, may often have some limitations due to disease differences (11). However, there are also some related research $(12,13)$ showing that the sensitivity and specificity of combined imaging diagnosis of malignant tumors are better than single detection. Therefore, CEUS combined with CT is proposed for the diagnosis of liver cancer. By retrospective analysis of 426 patients with liver cancer, the imaging performances were compared and their clinical values were analyzed. The aim is to provide reference and guidance for patients with liver cancer in future clinical trials.

CT makes a more accurate assessment of the location, number, size of lesions in the liver and the surrounding tissue 
of the lesion, and the tissue characteristics inside the lesion can be presented by enhanced scanning (13). According to the experimental results of Farinati et al (14), the difference between the intensified levels of CT examination of each stage can be used as an indicator of the source of blood supply, suggesting that CT can be used as a detection method of liver cancer metastasis. However, CEUS is a new imaging diagnostic technique in clinical practice and has high ability to distinguish blood dynamic changes in malignant tumor tissues (15). CEUS is better than CT in echogenic changes in lesion area, and the detection is more economical. The main component of contrast medium used in CEUS detection is SF6 gas microbubbles, which is more applicable for patients with cardiovascular disorders, not prone to iodine reaction (16). In this study, CEUS sensitivity and specificity for the diagnosis of liver cancer were 76.8 and $78.9 \%$. The sensitivity and specificity of CT were 81.2 and $85.5 \%$, respectively. The sensitivity and specificity of CEUS and CT were 90.4 and $92.7 \%$, respectively. The reason may be that the ultrasound microbubbles of CEUS detection can only be detected in the capillaries and blood pool, but not clearly in the extracellular space (17). Compared with CT, CEUS avoids the occurrence of misdiagnosis of peaks at intensified times and it shows real-time performance of overall liver lesions through dynamic performance (18). However, due to the weak ability of CEUS to detect lesions of atypical small hepatocellular carcinoma, the detection of differentiation and pathology of liver cancer is weaker than that of CT (19). CT scan reflects the characteristics of the lesions through the enhanced scanning of the liver and the use of liver function and cancer cell blood supply (20). CT can accurately show the situation of hepatic artery and blood vessels of patients, and the performance for hepatic arteriovenous fistula was significantly better than CEUS, CT also has better detection ability for small liver cancer (20). Therefore, CEUS more accurately show the 'whole' of liver cancer, CT is more subtle for the 'details'. The use of combined detection between the two makes up for each other's deficiencies, and provides higher accuracy of natural diagnosis. The results of Mauri et al (21) showed that the combined detection of CT and CEUS was superior to single imaging in the diagnosis of gastric cancer. This also proves the experimental results from the side.

In this study, through analyzing the $\mathrm{CT}$ and CEUS imaging results of 426 cases of liver cancer, it was proposed that $\mathrm{CT}$ combined with CEUS detection is more applicable for liver cancer. However, due to the lack of samples and more detailed analysis of the liver cancer type, we will conduct a more comprehensive analysis in future experiments.

In conclusion, CEUS combined with $\mathrm{CT}$ detection can make up for deficiencies in each other, effectively improving the diagnostic accuracy of liver cancer, which can be used as an effective detection method of liver cancer diagnosis.

\section{Acknowledgements}

Not applicable.

\section{Funding}

No funding was received.

\section{Availability of data and materials}

The datasets used and/or analyzed during the present study are available from the corresponding author on reasonable request.

\section{Authors' contributions}

BS contributed to the conception and design of this study. YL and DX interpreted the data and drafted this manuscript. JL collected the data and revised the manuscript. All authors read and approved the final manuscript.

\section{Ethics approval and consent to participate}

The study was approved by the Ethics Committee of Yuhuangding Hospital (Yantai, China). Signed written informed consents were obtained from the patients and/or guardians.

\section{Consent for publication}

Not applicable.

\section{Competing interests}

The authors have no conflicts of interest to declare.

\section{References}

1. Mignogna MD, Fedele S and Lo Russo L: The World Cancer Report and the burden of oral cancer. Eur J Cancer Prev 13: 139-142, 2004.

2. Kudo M: Surveillance, diagnosis, treatment, and outcome of liver cancer in Japan. Liver Cancer 4: 39-50, 2015.

3. Ryerson AB, Eheman CR, Altekruse SF, Ward JW, Jemal A, Sherman RL, Henley SJ, Holtzman D, Lake A, Noone AM, et al: Annual Report to the Nation on the Status of Cancer, 1975-2012, featuring the increasing incidence of liver cancer. Cancer 122: 1312-1337, 2016.

4. Chaubal N, Joshi M, Bam A and Chaubal R: Contrast-enhanced ultrasound of focal liver lesions. Semin Roentgenol 51: 334-357, 2016.

5. Albrecht MH, Scholtz JE, Kraft J, Bauer RW, Kaup M, Dewes P, Bucher AM, Burck I, Wagenblast J, Lehnert T, et al: Assessment of an advanced monoenergetic reconstruction technique in dual-energy computed tomography of head and neck cancer. Eur Radiol 25: 2493-2501, 2015.

6. Kudo M: Locoregional therapy for hepatocellular carcinoma. Liver Cancer 4: 163-164, 2015.

7. Shady W, Petre EN, Gonen M, Erinjeri JP, Brown KT, Covey AM, Alago W, Durack JC, Maybody M, Brody LA, et al: Percutaneous radiofrequency ablation of colorectal cancer liver metastases: Factors affecting outcomes - a 10-year experience at a single center. Radiology 278: 601-611, 2016.

8. Wildner D, Bernatik T, Greis C, Seitz K, Neurath MF and Strobel D: CEUS in hepatocellular carcinoma and intrahepatic cholangiocellular carcinoma in 320 patients - early or late washout matters: A subanalysis of the DEGUM multicenter trial. Ultraschall Med 36: 132-139, 2015.

9. Fischer MA, Kartalis N, Grigoriadis A, Loizou L, Stål P, Leidner B, Aspelin P and Brismar TB: Perfusion computed tomography for detection of hepatocellular carcinoma in patients with liver cirrhosis. Eur Radiol 25: 3123-3132, 2015.

10. Mishima M, Toh U, Iwakuma N, Takenaka M, Furukawa M and Akagi Y: Evaluation of contrast Sonazoid-enhanced ultrasonography for the detection of hepatic metastases in breast cancer. Breast Cancer 23: 231-241, 2016.

11. Wells ML, Venkatesh SK, Chandan VS, Fidler JL, Fletcher JG, Johnson GB, Hough DM and Roberts LR: Biphenotypic hepatic tumors: Imaging findings and review of literature. Abdom Imaging 40: 2293-2305, 2015. 
12. Minami Y and Kudo M: Imaging modalities for assessment of treatment response to nonsurgical hepatocellular carcinoma therapy: Contrast-enhanced US, CT, and MRI. Liver Cancer 4: $106-114,2015$.

13. Lee YJ, Lee JM, Lee JS, Lee HY, Park BH, Kim YH, Han JK and Choi BI: Hepatocellular carcinoma: Diagnostic performance of multidetector CT and MR imaging - a systematic review and meta-analysis. Radiology 275: 97-109, 2015.

14. Farinati F, Vanin V, Giacomin A, Pozzan C, Cillo U, Vitale A, Di Nolfo AM, Del Poggio P, Benvegnu L, Rapaccini G, et al Italian Liver Cancer (ITA.LI.CA) group: BCLC stage B hepatocellular carcinoma and transcatheter arterial chemoembolization: A 20 -year survey by the Italian Liver Cancer group. Liver Int 35 223-231, 2015.

15. Bartolotta TV, Vernuccio F, Taibbi A and Lagalla R: Contrast-enhanced ultrasound in focal liver lesions: Where do we stand? Semin Ultrasound CT MR 37: 573-586, 2016.

16. Fröhlich E, Muller R, Cui XW, Schreiber-Dietrich D and Dietrich CF: Dynamic contrast-enhanced ultrasound for quantification of tissue perfusion. J Ultrasound Med 34: 179-196, 2015

17. Nishigaki Y, Hayashi H, Tomita E, Suzuki Y, Watanabe N, Watanabe S, Watanabe C, Takagi Y, Kato T and Naiki T: Usefulness of contrast-enhanced ultrasonography using Sonazoid for the assessment of therapeutic response to percutaneous radiofrequency ablation for hepatocellular carcinoma. Hepatol Res 45 : 432-440, 2015
18. Li R, Yang D, Tang CL, Cai P, Ma KS, Ding SY, Zhang XH, Guo DY and Yan XC: Combined hepatocellular carcinoma and cholangiocarcinoma (biphenotypic) tumors: Clinical characteristics, imaging features of contrast-enhanced ultrasound and computed tomography. BMC Cancer 16: 158, 2016.

19. Kudo M, Matsui O, Izumi N, Iijima H, Kadoya M, Imai Y, Okusaka T, Miyayama S, Tsuchiya K, Ueshima K, et al; Liver Cancer Study Group of Japan: JSH consensus-based clinical practice guidelines for the management of hepatocellular carcinoma: 2014 update by the Liver Cancer Study Group of Japan. Liver Cancer 3: 458-468, 2014.

20. Tacher V, Radaelli A, Lin M and Geschwind JF: How I do it: Cone-beam CT during transarterial chemoembolization for liver cancer. Radiology 274: 320-334, 2015.

21. Mauri G, Cova L, De Beni S, Ierace T, Tondolo T, Cerri A, Goldberg SN and Solbiati L: Real-time US-CT/MRI image fusion for guidance of thermal ablation of liver tumors undetectable with US: Results in 295 cases. Cardiovasc Intervent Radiol 38: 143-151, 2015. International (CC BY-NC-ND 4.0) License. 